\section{Fourier-domain optical coherence tomography of eyes with idiopathic epiretinal membrane: correlation between macular morphology and visual function}

S Pilli, P Lim, RJ Zawadzki, SS Choi, JS Werner and SS Park and/or Verhoeff's membrane, vitreal displacement of foveal outer nuclear layer and foveal detachment $(P<0.05)$. Foveal IS-OS junction disruption was seen in $25 \%$ of eyes on Stratus OCT but in none of the eyes on FD-OCT.

Conclusion FD-OCT allowed improved visualization of ERM and associated foveal morphological changes that correlated best with BCVA. Macular thickening correlated weakly with decreased macular function as assessed by MP-1.

Eye (2011) 25, 775-783; doi:10.1038/eye.2011.55; published online 25 March 2011

Keywords: epiretinal membrane;

Fourier-domain optical coherence tomography; macular morphology; Stratus OCT; microperimetry

Introduction

Epiretinal membrane (ERM) is a fibrocellular membrane seen on the inner retinal surface in the macular area after partial or complete posterior vitreous detachment. ${ }^{1}$ ERM can be idiopathic or secondary to trauma, surgery, ocular inflammation and other causes. ${ }^{2}$ Often patients are asymptomatic with near normal vision, but at times ERM could lead to significant metamorphopsia and vision loss. The vision loss is speculated to result from factors such as full thickness retinal folds, foveal elevation, foveal ectopia, presence of opaque membrane directly over the fovea or from direct
Department of

Ophthalmology \& Vision Science, University of

California Davis Eye Center, Sacramento, CA, USA

Correspondence: SS Park, Department of Ophthalmology and Vision Science, University of California, Davis Eye Center 4860 Y Street, Suite 2400, Sacramento, CA 95817, USA

Tel: + 1916734 6544; Fax: + 19167346197 E-mail: susanna.park@ ucdmc.ucdavis.edu

Received: 8 July 2010 Accepted in revised form: 8 February 2011 Published online: 25 March 2011

Meeting presentation: Presented in part as a poster at the Retina Congress, Vancouver, August, 2010 and presented in part as a poster at the annual meeting of the American Academy of Ophthalmology, New Orleans, LA, 2007. 
traction on the fovea. ${ }^{2}$ However, these gross macular structural changes do not always correlate with the degree of vision loss.

Since the introduction of optical coherence tomography (OCT) to clinical practice, some studies have reported a correlation between an increase in central macular thickness (CMT) on time-domain OCT with loss of visual acuity associated with ERM preoperatively, but increase in CMT does not always result in vision loss. In addition, the correlation between CMT and visual acuity was not noted postoperatively after vitrectomy surgery. ${ }^{4-6}$ Multifocal ERG studies showed changes in both the inner and outer retinal layers associated with ERM leading to speculations that changes in both inner and outer retinal layers may account for the decrease in visual function associated with idiopathic ERM. ${ }^{7}$

Fourier-domain OCT (FD-OCT) is a novel extension of OCT technology, in which the sample structure is measured in the form of spectral fringes and the inverse Fourier transformation is used to extract the depth scattering intensity profile (A-scan) ${ }^{8}$ The parallel A-scan detection achieved with FD-OCT allows for a 100-fold higher data acquisition speed over time-domain (TD-OCT) without reduction in system sensitivity, leading to real time, in vivo imaging with fewer motion artifacts. ${ }^{9}$ The improved axial resolution because of the broader spectral band width of the light source allows better visualization of the intraretinal structures, especially the external limiting membrane and inner segment-outer segment (IS-OS) junction of the photoreceptor layer. ${ }^{10}$ In this study, we used a researchgrade high-resolution FD-OCT system to study the macular morphological and volumetric changes in the retinal layers associated with idiopathic ERM and to correlate these changes with visual function as assessed by visual acuity and microperimetry (MP-1).

\section{Materials and methods}

All patients enrolled in this prospective observational case series were evaluated at the Retina Service University of California Davis Eye Center from August 2006 to October 2009. The study was conducted under the tenets of Declaration of Helsinki. A written informed consent was obtained from all the participants. The sole inclusion criterion was clinically identified idiopathic ERM, both symptomatic and asymptomatic. Patients were excluded if they had secondary ERM (eg, history of retinal detachment surgery, uveitis and so on) or if they had any other ocular pathology that could interfere with visual function (eg, other concurrent maculopathy, visually significant cataracts and so on).

All subjects underwent a comprehensive eye examination, including ETDRS best-refracted visual acuity
(BCVA) at $4 \mathrm{~m}$ and a dilated fundus examination. Stratus OCT imaging was carried out in 20 eyes of 15 subjects and included the fast macular map and six radial line scans. The thickness of the central $1 \mathrm{~mm}$ zone of the ETDRS macular thickness map obtained from the fast macular map on Stratus OCT was defined as CMT in this study. Radial line scan with signal strength $\geqslant 6$ was converted to gray scale and was analyzed for the presence of the ERM and morphological changes in the IS-OS junction.

A research-grade FD-OCT system developed at the University of California Davis, Vision Science and Advanced Retinal Imaging Laboratory with an axial and transverse resolution of $4.5 \mu \mathrm{m}$ and 10 to $15 \mu \mathrm{m}$,

respectively, was used to image maculae of all 24 eyes of 19 subjects. ${ }^{9}$ The system acquisition speed ranged from 9000 lines/s (9 frames/s; 1000 A-scans/frame) to 23000 lines/s (23 frames/s) during the study period. A standard imaging session included acquisition of a video of $6 \mathrm{~mm}$ horizontal line scans and at least two volumetric data sets covering $6 \times 6 \mathrm{~mm}$ and $5 \times 5 \mathrm{~mm}$ area of the retina (this resulted in $6 \times 6 \times 2$ and $5 \times 5 \times 2$ (lateral $\times$ lateral $\times$ depth)) volumes. Each volume was acquired using a raster scan consisting of 100 B-scans with 1000 A-scans per frame and each B scan was separated by $60 \mu \mathrm{m}$ for $6 \times 6$ scans and $50 \mu \mathrm{m}$ for $5 \times 5$ scans. Small motion shifts between consecutive B-scans were corrected using a frame cross-correlation-based algorithm. ${ }^{11}$

To correlate BCVA with macular morphological changes seen on FD-OCT, the eyes with ERM were divided into three groups based on BCVA: group 1 with BCVA: $\geq 20 / 20(N=6)$, group II with BCVA: $20 / 25$ to 20/32 $(N=9)$ and group III with BCVA: $\leq 20 / 40(N=9)$. One hundred serial B-scans were analyzed for the presence of morphological changes in the inner retinal layers (ie, layers superficial to the outer nuclear (ONL)), outer retinal layers (ie, ONL and deeper layers), foveal photoreceptor IS-OS junction and Verhoeff's membrane (VM), foveal contour, and the pattern of adherence of the ERM to the inner retina. The inner retinal layers were evaluated for tethering of the nerve fiber layer (NFL), striae (or disarrangement) of the inner retinal layers, and wrinkling or irregularities on the inner retinal surface. The outer retinal layers were evaluated for tenting or vitreal displacement of the foveal ONL, and striae of the photoreceptor IS-OS junction. The foveal photoreceptor IS-OS junction and VM were evaluated for increase in convexity of the IS-OS junction, blurring of the IS/OS junction and/or VM. Pseudohole on FD-OCT was identified by a steep foveal pit with thickened edges and lamellar hole was identified by an irregular, thinned foveal floor with split in foveal edges on the most centered B-scan. ${ }^{12}$ For morphological evaluation, an FD-OCT B-scan from a normal eye was used as a reference image. ${ }^{13}$ 
Central foveal thickness (CFT) was measured manually by placing the calipers from the inner retinal surface to the retinal pigment epithelial layer at the center of the fovea on the most centered FD-OCT B-scan, using NIH Image-J software. ${ }^{14}$ Macular volume (MV) measurement of the central $3 \mathrm{~mm}$ area was obtained by segmenting retinal structures using a support vector machine (SVM)-based segmentation algorithm (volume visualization and image processing software) and processing the thickness maps in LabVIEW image processing software developed by VSRI, Department of Ophthalmology \& Vision Science, University of Davis Eye Center California, using the LabVIEW programming language (National Instrument, Austin, TX, USA) as described by Zawadzki et al. ${ }^{11}$

MP-1 testing was carried out using microperimeter (MP-1; Nidek Technologies, Padova, Italy) in 15 eyes of 11 subjects. The central $16^{\circ}$ was tested with an undilated pupil; Goldmann size III target and 4-2-1 staircase strategy were used. Mean MP score was determined by calculating the average of 46 points in the central $12^{\circ}$ and was correlated with the total $\mathrm{MV}$ in the central $3 \mathrm{~mm}$ area obtained from the FD-OCT volume scans $\left(1.25^{\circ}\right.$ from the fovea is equivalent to $0.35 \mathrm{~mm}$ from the fovea). ${ }^{15}$ The MP-1 test was considered reliable if $>80 \%$ of fixation points were within $2^{\circ}$ and $90 \%$ of fixation points were within $4^{\circ}$ of center. For qualitative comparison of macular thickness maps with MP-1 sensitivity, we overlaid numerical MP-1 data on the fundus image over the retinal thickness map with retinal vessels and foveal center acting as reference points, as carried out previously. ${ }^{16}$

\section{Statistical analysis}

ETDRS BCVA was converted to log of the minimum angle of resolution (logMAR) for statistical analysis. Correlation between mean logMAR BCVA and macular thickness or volume was analyzed using the Pearson's correlation coefficient. To evaluate the features seen on FD-OCT among the three groups an ANOVA single-factor analysis was carried out to compare the non-categorical data (CFT, $\mathrm{MV})$ and the $\chi^{2}$-test was used to compare the categorical data (morphological changes in macular layers). A $P$-value of $<0.05$ was considered significant.

\section{Results}

Table 1 summarizes the demographic data of the 24 eyes from 19 subjects enrolled in this study and imaged with FD-OCT. Mean age was $72 \pm 7.01$ years (range: $57-84$ years). We had 11 (58\%) female subjects. Mean logMAR BCVA was $0.18 \pm 0.16$ (range: -0.08 to 0.48 ) and mean ETDRS BCVA Snellen equivalent was $20 / 32$ (range: $20 / 15^{-1}$ to $20 / 60$ ).

Of these 24 eyes with iERM diagnosed clinically, ERM could be visualized with FD-OCT in all 24 eyes (100\%). In contrast, ERM was visualized in $17(85 \%)$ of the 20
Table 1 Summary of demographic data, visual acuity, retinal thickness and volume measurement, and microperimetry data of all eyes with idiopathic epiretinal membrane imaged using Stratus and high-resolution Fourier-domain optical coherence tomography

\begin{tabular}{lc}
\hline Number of eyes (subjects) & $24(19)$ \\
Age, years (mean \pm SD; range) & $72 \pm 7.01(57-84)$ \\
Gender (number/\%) & $8(42)$ \\
$\quad$ Men & $11(58)$ \\
Women & \\
Eye (number/\%) & $12(50)$ \\
$\quad$ Right & $12(50)$ \\
Left & $20 / 32 \pm 12.86 ;$ \\
ETDRS BCVA (mean $\pm S D ;$ range) & $\left(20 / 15^{-1}-20 / 60\right)$ \\
& $0.18 \pm 0.16 ;$ \\
LogMAR BCVA (mean $\pm S D ;$ range) & $(-0.08-0.48)$ \\
& $329.75 \pm 90.85 ;$ \\
CMT Stratus OCT, $\mu$ m (mean $\pm S D ;$ range) & $(179-504)$ \\
& $360.60 \pm 130.44 ;$ \\
CFT FD-OCT, $\mu \mathrm{m}$ (mean $\pm S D ;$ range) & $(170-583)$ \\
& $2.86 \pm 0.38 ;$ \\
MV FD-OCT, mm ${ }^{3}$ (mean $\pm S D ;$ range) & $(2.36-3.71)$ \\
& $18.83 \pm 1.09 ;$ \\
Microperimetry, dB (mean $\pm S D ;$ range) & $(17.3-20)$ \\
&
\end{tabular}

Abbreviations: BCVA, best-refracted ETDRS visual acuity; CMT, central macular thickness for central $1 \mathrm{~mm}$; CFT, central foveal thickness; FD, Fourier-domain; logMAR, log of the minimum angle of resolution; $\mathrm{MV}$, macular volume for central $3 \mathrm{~mm}$; OCT, optical coherence tomography.

eyes (15 subjects) imaged on Stratus OCT. With regard to foveal contour, FD-OCT revealed a normal foveal depression in $3(12.5 \%)$ eyes, pseudohole in 2 (8.33\%) eyes, lamellar hole in $3(12.5 \%)$ eyes, foveal depression with macular thickening in $3(12.5 \%)$ eyes, and partial to complete loss of foveal depression with macular thickening in 13 (54.16\%) eyes.

Figure 1 shows the correlation between logMAR BCVA and CMT on Stratus OCT and FD-OCT CFT and MV (MV for central $3 \mathrm{~mm}$ area). Mean CMT for the central $1 \mathrm{~mm}$ area of the 20 eyes imaged with Stratus OCT was $329.75 \pm 90.85 \mu \mathrm{m}$ (range: 179 to $504 \mu \mathrm{m}$ ). CMT on Stratus OCT correlated positively with logMAR BCVA $(r=0.70$, $P=0.0005)$. LogMAR BCVA also correlated with MV on FD-OCT for the central $3 \mathrm{~mm}$ area (mean $2.86 \pm 0.38 \mathrm{~mm}^{3}$, range: 2.36 to $\left.3.71 \mathrm{~mm}^{3} ; r=0.71, P=<0.0001\right)$, but the best correlation was with CFT on FD-OCT (mean $360.61 \pm$ $130.44 \mu \mathrm{m}$, range: 170 to $583 \mu \mathrm{m} ; r=0.83, P<0.0001$ ).

\section{Correlation between the BCVA and macular morphological changes}

Table 2 summarizes the findings of the 24 eyes imaged with FD-OCT separated into three groups based on 

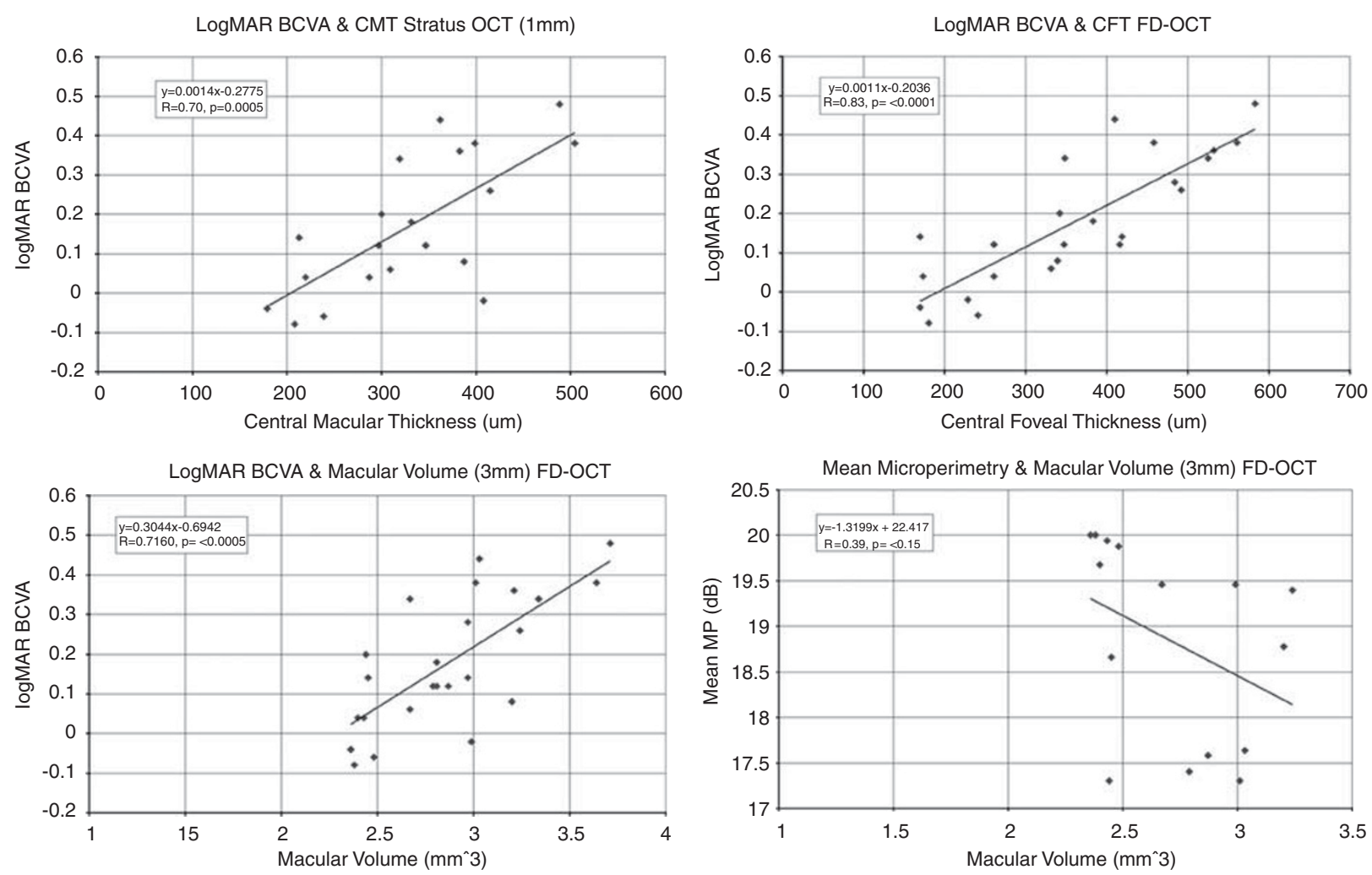

Figure 1 Scatter plots correlating the logMAR best-corrected visual acuity (BCVA) with central macular thickness (CMT), central foveal thickness (CFT), and macular volume (MV) and mean microperimetry (MP-1) sensitivity to total MV. Scatter plots showing correlations between the logMAR BCVA and CMT (central $1 \mathrm{~mm}$ area of macula) on Stratus optical coherence tomography (OCT), CFT and total MV (central $3 \mathrm{~mm}$ macular area) on high-resolution Fourier-domain (FD)-OCT in eyes with idiopatic epiretinal membrane. No significant correlation is noted between mean MP-1 sensitivity and total MV for central $3 \mathrm{~mm}$ area on FD-OCT in eyes with idiopathic epiretinal membrane (bottom, left).

BCVA. Group I had six eyes with logMAR BCVA ranging from -0.08 to 0.04 (Snellen equivalent BCVA range: $20 / 15^{-1}$ to $20 / 20$ ). For this group, mean CMT on Stratus OCT was $256.83 \pm 82.31 \mu \mathrm{m}$ (range: 179 to $408 \mu \mathrm{m}$ ) and mean CFT on FD-OCT was $209 \pm 39.46 \mu \mathrm{m}$ (range: 170 to $261 \mu \mathrm{m}$ ). Mean MV on FD-OCT was $2.50 \pm 0.24 \mathrm{~mm}^{3}$ (range: 2.36 to $2.99 \mathrm{~mm}^{3}$ ). The large difference in ranges seen for CMT and CFT in group I was mainly because of the one eye with BCVA of 20/20 that had massive cystoid macular edema (CME) on Stratus OCT with CMT of $408 \mu \mathrm{m}$. The FD-OCT B-scan image through the fovea of this eye showed an area of subtle dehiscence over an apparent central pseudocyst, suggestive of a lamellar hole formation with ERM (Figure 2a). Thus, the CFT of $229 \mu \mathrm{m}$, by FDOCT was much lower than the CMT that correlated better with BCVA. The remaining eyes in group I had no foveal thickening or morphological changes within the retinal layers in the macula except for mild wrinkling of the inner retinal surface seen in three eyes.

Group II had nine eyes with $\log M A R$ BCVA ranging from 0.06 to 0.2 (Snellen equivalent BCVA range: $20 / 25$ to 20/32). Mean CMT on Stratus OCT was $312 \pm 53.83 \mu \mathrm{m}$ (range: 213 to $387 \mu \mathrm{m}$ ) and mean CFT on FD-OCT was
$334 \pm 78.08 \mu \mathrm{m}$ (range: 170 to $418.6 \mu \mathrm{m}$ ). Mean MV on FD-OCT was $2.77 \pm 0.23 \mathrm{~mm}^{3}$ (range: 2.44 to $3.20 \mathrm{~mm}^{3}$ ). Seven of nine eyes in Group II had subtle to prominent vitreal displacement of the foveal ONL; five eyes had partial to total loss of foveal depression with macular thickening, and six eyes had either increase in the convexity of the foveal photoreceptor IS-OS and/or blurring of the VM and foveal photoreceptor IS-OS (Figure 2b). Foveal detachment was seen in two eyes. One of them also had tethering of the NFL.

Group III had nine eyes with $\log$ MAR BCVA ranging from 0.26 to 0.48 (Snellen equivalent BCVA range: $20 / 40$ to 20/60). Mean CMT on Stratus OCT was $410 \pm 66.30 \mu \mathrm{m}$ (range: 319 to $504 \mu \mathrm{m}$ ) and mean CFT on FD-OCT was $488 \pm 74.39 \mu \mathrm{m}$ (range: 348 to $583 \mu \mathrm{m})$. Mean MV on FD-OCT was $3.20 \pm 0.33 \mathrm{~mm}^{3}$ (range: 2.67 to $3.71 \mathrm{~mm}^{3}$ ). Eight eyes in this group $(89 \%)$ had a massive macular thickening with prominent striae of the inner retinal layers, vitreal displacement of foveal ONL and loss of foveal depression with bridging ERM over the fovea (Figure 2c). Four eyes had an increase in the convexity of the foveal photoreceptor IS-OS junction with blurring of the VM, whereas two 
Table 2 Summary of morphological changes noted on high-resolution Fourier-domain optical coherence tomography in eyes with idiopathic epiretinal membrane classified based on best-corrected visual acuity

\begin{tabular}{|c|c|c|c|}
\hline Number of eyes & $\begin{array}{l}\text { Group I } \\
6\end{array}$ & $\begin{array}{l}\text { Group II } \\
9\end{array}$ & $\begin{array}{l}\text { Group III } \\
9\end{array}$ \\
\hline $\begin{array}{l}\mathrm{LogMAR} B C V A, \text { mean } \pm \mathrm{SD} \\
\text { (range); (Snellen equivalent, } \\
\text { range) }\end{array}$ & $\begin{array}{l}-0.02 \pm 0.05(-0.08 \text { to } 0.04) \\
\left(20 / 15^{-1} \text { to } 20 / 20\right)\end{array}$ & $\begin{array}{l}0.13 \pm 0.04(0.06 \text { to } 0.20) \\
(20 / 25 \text { to } 20 / 32)\end{array}$ & $\begin{array}{l}0.36 \pm 0.07(0.26 \text { to } 0.48) \\
(20 / 40 \text { to } 20 / 60)\end{array}$ \\
\hline $\begin{array}{l}\mathrm{CFT}(\mu \mathrm{m}) \text {, mean } \pm \mathrm{SD} \\
\text { (range) }\end{array}$ & $209 \pm 39.46$ (170 to 261$)$ & $334 \pm 78.08$ (170 to 418.6$)$ & $488.11 \pm 74.39(348$ to 583$)$ \\
\hline $\begin{array}{l}\text { Stratus OCT CMT }(\mu \mathrm{m}) \text {, } \\
\text { mean } \pm \text { SD (range) }\end{array}$ & $\begin{array}{l}256.83 \pm 82.31 \text { (179 to } 408 ; \\
n=6)\end{array}$ & $312 \pm 53.83(213$ to $387 ; n=7)$ & $410 \pm 66.30,(319$ to $504 ; n=7)$ \\
\hline $\begin{array}{l}\mathrm{MV}\left(\mathrm{mm}^{3}\right) \text {, mean } \pm \mathrm{SD} \\
\text { (range) }\end{array}$ & $2.50 \pm 0.24(2.36$ to 2.99$)$ & $2.77 \pm 0.23$ (2.44 to 3.20$)$ & $3.20 \pm 0.33(2.67$ to 3.71$)$ \\
\hline Foveal contour & $\begin{array}{l}\text { 1. Normal: } N=4 \\
\text { 2. Lamellar hole: } N=1 \\
\text { 3. Pseudohole: } N=1 \\
\text { 4. Partial to total loss of foveal } \\
\text { depression with retinal } \\
\text { thickening: } N=0\end{array}$ & $\begin{array}{l}\text { 1. Normal: } N=2 \\
\text { 2. Lamellar hole: } N=1 \\
\text { 3. Pseudohole: } N=1 \\
\text { 4. Partial to total loss of foveal } \\
\text { depression with retinal } \\
\text { thickening: } N=5\end{array}$ & $\begin{array}{l}\text { 1. Normal: } N=0 \\
\text { 2. Lamellar hole: } N=1 \\
\text { 3. Pseudohole: } N=0 \\
\text { 4. Partial to total loss of foveal } \\
\text { depression with retinal } \\
\text { thickening: } N=8\end{array}$ \\
\hline $\begin{array}{l}\text { Foveal morphological } \\
\text { changes }\end{array}$ & $\begin{array}{l}\text { 1. Normal: } N=5 \\
\text { 2. Increased convexity with } \\
\text { blurring of VM: } N=0 \\
\text { 3. Blurring of IS-OS and VM: } \\
N=0 \\
\text { 4. Vitreal displacement of } \\
\text { foveal ONL: } N=1 \\
\text { 5. Foveal detachment: } N=0\end{array}$ & $\begin{array}{l}\text { 1. Normal: } N=1 \\
\text { 2. Increased convexity with } \\
\text { blurring of VM: } N=3 \\
\text { 3. Blurring of IS-OS and VM: } \\
N=3 \\
\text { 4. Vitreal displacement of } \\
\text { foveal ONL: } N=7 \\
\text { 5. Foveal detachment: } N=2\end{array}$ & $\begin{array}{l}\text { 1. Normal: } N=1 \\
\text { 2. Increased convexity with } \\
\text { blurring of VM: } N=4 \\
\text { 3. Blurring of IS-OS and VM: } \\
N=2 \\
\text { 4. Vitreal displacement of fovea } \\
\text { ONL: } N=8 \\
\text { 5. Foveal detachment: } N=3\end{array}$ \\
\hline $\begin{array}{l}\text { Extrafoveal morphological } \\
\text { changes in retinal layers }\end{array}$ & $\begin{array}{l}\text { 1. Normal: } N=3 \\
\text { 2. Inner retinal changes: } \\
\text { (a) Tethering of the NFL: } \\
N=0 \\
\text { (b) Striae within inner } \\
\text { retinal layers: } N=1 \\
\text { (c) Irregular inner retinal } \\
\text { surface: } N=3 \\
\text { 3. Outer retinal changes } \\
- \text { Striae of IS-OS junction: } \\
N=0\end{array}$ & $\begin{array}{l}\text { 1. Normal: } N=1 \\
\text { 2. Inner retinal changes: } \\
\text { (a) Tethering of the NFL: } N=1 \\
\text { (b) Striae within inner retinal } \\
\text { layers: } N=4 \\
\text { (c) Irregular inner retinal } \\
\text { surface: } N=8 \\
\text { 3. Outer retinal changes } \\
\text { - Striae of the IS-OS junction: } \\
N=0\end{array}$ & $\begin{array}{l}\text { 1. Normal: } N=0 \\
\text { 2. Inner retinal changes: } \\
\text { (a) Tethering of the NFL: } N=5 \\
\text { (b) Striae within inner retinal } \\
\text { layers: } N=9 \\
\text { (c) Irregular inner retinal } \\
\text { surface: } N=9 \\
\text { 3. Outer retinal changes } \\
\text { - Striae of the IS-OS junction: } \\
N=1\end{array}$ \\
\hline
\end{tabular}

Abbreviations: BCVA, best-corrected visual acuity; CFT, central foveal thickness; CMT, central macular thickness; FD-OCT, high-resolution Fourierdomain optical coherence tomography; IS-OS, inner segment-outer segment junction of photoreceptor layer; $\log$ MAR, log of the minimum angle of resolution; MV, macular volume for central $3 \mathrm{~mm}$; NFL, nerve fiber layer; ONL, outer nuclear layer; VM, Verhoeff's membrane.

eyes had blurring of the foveal photoreceptor IS-OS junction and VM. Tethering of the NFL was seen in five eyes (Figure 2d). Foveal detachment was seen in three eyes. The photoreceptor IS-OS junction was clearly visualized in all eyes outside the foveal area, with striae of this layer noted in one eye outside the fovea.

Comparison of the morphological features among the three groups noted on FD-OCT using an ANOVA single-factor analysis showed significant differences for CFT $(P<0.0001)$ and MV $(P=0.0003)$. A $\chi^{2}$ analysis for the categorical data seen between the three groups showed statistically significant difference in morphological changes in the fovea including vitreal displacement of ONL, blurring of foveal IS-OS junction and/or VM, change in convexity of foveal IS-OS function and foveal detachment $\left(\chi^{2}\right.$ with Yates correction for continuity $=7.462$, d.f. $=2$, $P=0.023)$. Among extrafoveal morphological changes noted on FD-OCT, striae of the inner retinal layers was the only change that showed a significant difference in incidence among the three groups ( $\chi^{2}$ with Yates correction for continuity $=7.829$, d.f. $=2, P=0.019$ ). No statistically significant difference was noted in incidence of irregular inner retinal surface $(P=0.17)$, the tethering of the NFL $(P=0.12)$ and the changes (loss) in the foveal depression $(P=0.066)$, although these features were more common in eyes with poorer vision. 

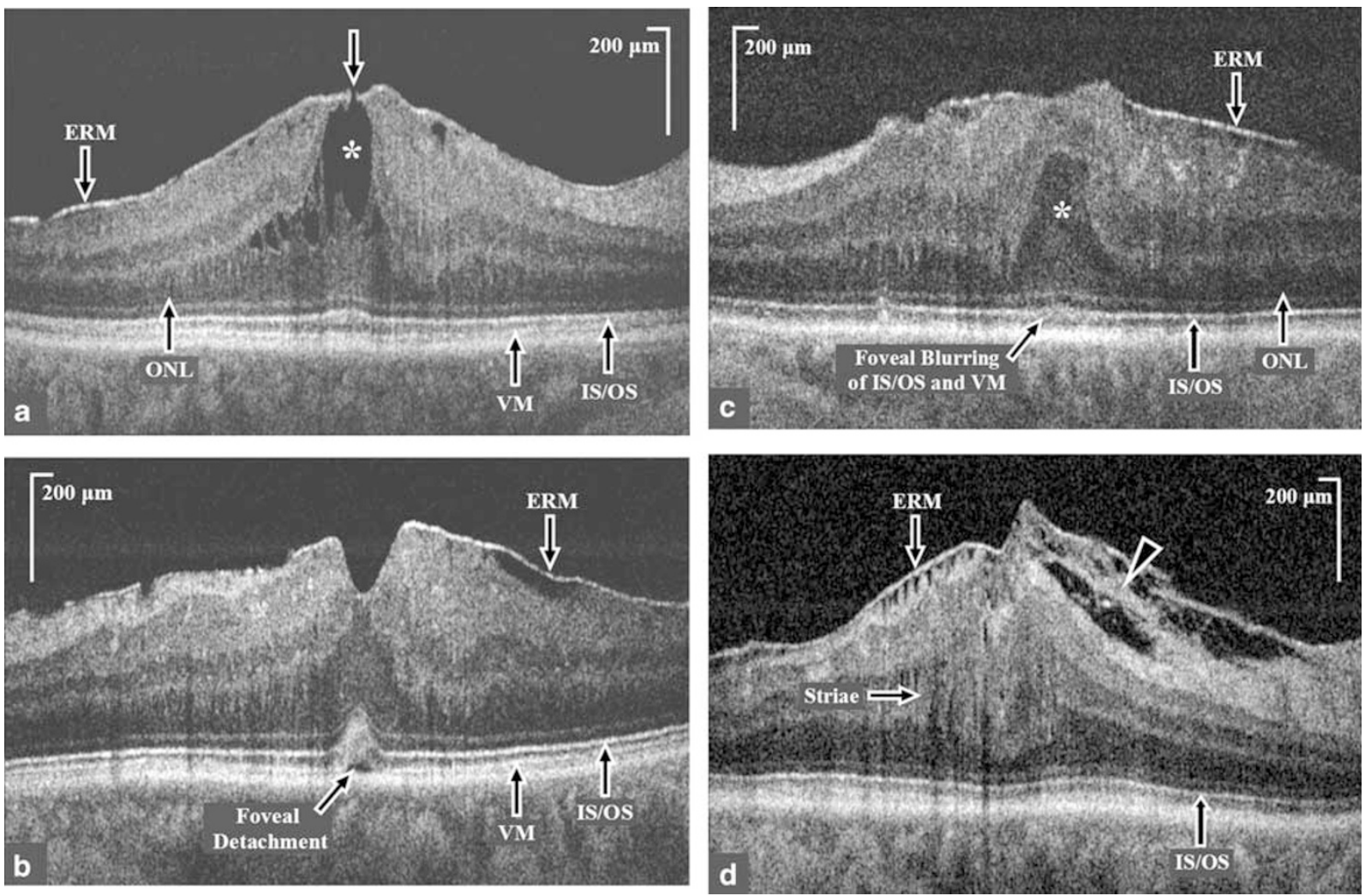

Figure 2 High-resolution Fourier-domain optical coherence tomography (FD-OCT) B-scan macular images through the fovea of eyes with idiopathic epiretinal membrane (ERM). (a) High-resolution FD-OCT image through the fovea of an eye with BCVA of 20/20, (group 1) showing a lamellar hole (white arrow) with cystoid spaces (white asterisk) in the outer nuclear layer (ONL), and a normal inner segment-outer segment (IS/OS) junction of the photoreceptor layer. Central foveal thickness was $229 \mu \mathrm{m}$ on FD-OCT, whereas central macular thickness on Stratus OCT was $408 \mu \mathrm{m}$. (b) High-resolution FD-OCT image of an eye with BCVA of 20/25 (group II) showing partially intact foveal depression, an increased convexity and blurring of the foveal IS/OS junction and Verhoeff's membrane (VM) and a foveal detachment. (c) High-resolution FD-OCT image of an eye with BCVA of 20/50 showing the presence of epiretinal membrane (ERM), anterior herniation of the outer nuclear layer (ONL; white asterisk) at the fovea and blurring of the foveal IS/OS junction and VM. (d) High-resolution FD-OCT extra-foveal image of an eye with BCVA of 20/50 showing the presence of ERM with tethering of the nerve fiber layer (white arrow head) and striae of the inner retinal layers (white arrow) and a normal IS/OS junction.

\section{Comparison of IS-OS junction between Stratus OCT and FD-OCT}

We also compared the appearance of the foveal photoreceptor IS-OS junction between Stratus OCT and FD-OCT in 20 eyes that were imaged with both instruments. Mean signal strength of Stratus OCT images was $8.23 \pm 1.57$. On Stratus OCT, the IS-OS junction appeared normal in 15 eyes (75\%) and focal disruptions in the fovea were seen in 5 eyes (25\%), whereas FD-OCT showed an intact IS-OS junction line in all the B-scans of 24 eyes $(100 \%)$. This difference was significant $\left(\chi^{2}\right.$ with Yates correction for continuity $=3.657$, d.f. $=1, P=0.05)$.

\section{Correlation between MP-1 sensitivity and macular thickness}

In all, 15 eyes of 11 subjects had functional evaluation with MP-1. Mean $\log$ MAR BCVA was $0.11 \pm 0.15$ among these eyes. Mean MP-1 sensitivity covering the central $12^{\circ}$ was $18.83 \pm 1.09 \mathrm{~dB}$ (range: 17.3 to $20 \mathrm{~dB}$ ) which correlated poorly with MV (mean: $2.71 \pm 0.31 \mathrm{~mm}^{3}$, range: 2.36 to $3.24 \mathrm{~mm}^{3}$ ) for the corresponding central $3 \mathrm{~mm}$ area of the macula on FD-OCT $(r=0.38, P=0.15$; Figure $1 \mathrm{~d})$.

On comparing macular thickness maps from FD-OCT with macular sensitivity maps obtained using MP-1 for all 15 eyes, 10 eyes (67\%) showed some qualitative correlation, that is, regions of the macular with decreased sensitivity correlated with areas of the macula with more severe thickening (Figure 3). However, the remaining five eyes $(33 \%)$ showed poor or no correlation with the macular sensitivity map obtained using MP-1.

\section{Discussion}

This study used a research-grade high-resolution FD-OCT instrument to visualize ERM and study in detail the associated macular morphological changes that 

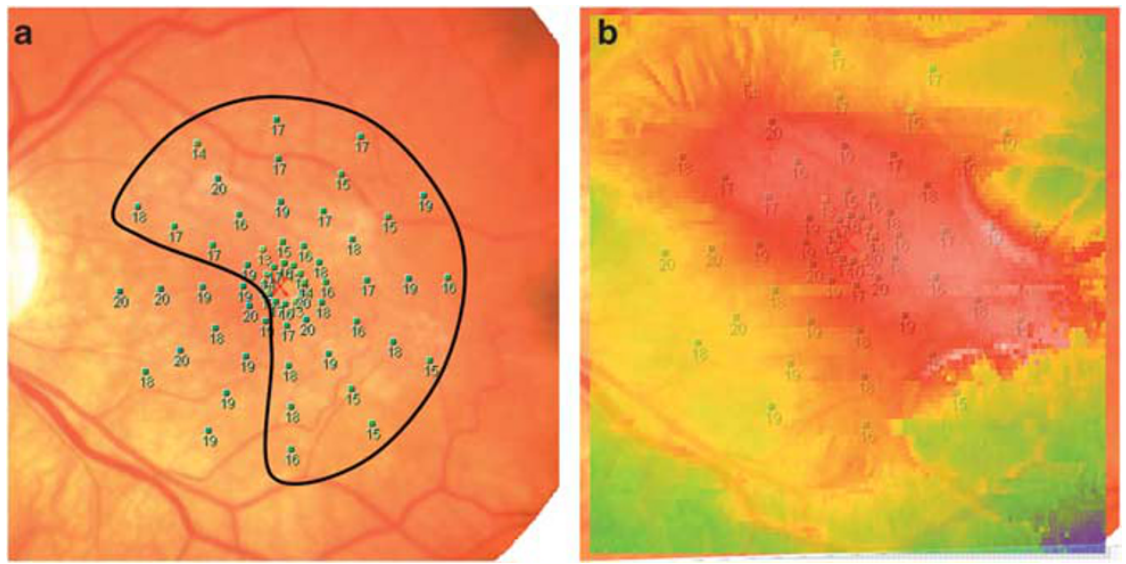

Figure 3 Correlation between change in microperimetry (MP-1) sensitivity and macular thickness map. (a) Numerical MP-1 data on color fundus image of an eye with idiopathic epiretinal membrane with BCVA of 20/50 showing diffuse moderate loss of sensitivity with sparing of the inferonasal macula. (b) Overlay of the MP-1 data on the color-coded macular thickness map showing a good qualitative correlation between the area of decreased MP-1 sensitivity and increased macular thickness (red color indicates more thickened macula area).

correlated with vision loss. In our series, high-resolution FD-OCT clearly identified the ERM in all eyes imaged. The sensitivity was higher than Stratus OCT, which detected ERM in only $85 \%$ of eyes in our series. Massin et $a l^{4}$ reported that ERM was visualized in only $42 \%$ of eyes with clinically diagnosed ERM using a TD-OCT instrument (Humphrey Company, San Leandro, CA, USA) with an axial resolution of $13 \mu \mathrm{m}$. The improved axial resolution and dense tissue sampling offered by FD-OCT enabled better detection of the ERM.

Our study demonstrates that FD-OCT also allowed detailed visualization of the morphological changes within the macula associated with traction that may result in vision loss. Although a strong correlation was noted between BCVA and CMT on Stratus OCT similar to previous reports, ${ }^{3,4}$ a stronger correlation was noted between CFT on FD-OCT and BCVA. This is because FD-OCT allowed identification of subtle changes in the fovea that may affect CMT measurement. As exemplified in Figure 2a, an asymptomatic eye with 20/20 vision and apparent massive CME on Stratus OCT actually had a central pseudocyst, which appeared to be a lamellar hole on FD-OCT. This may explain why some eyes with apparent macular edema on Stratus OCT may have minimal vision loss associated with ERM.

In our series, increase in CFT and changes in foveal morphology and contour on FD-OCT correlated the best with decrease in BCVA. As the inner retinal layers are lacking in the fovea, an increase in CFT would represent traction on the foveal photoreceptors. Loss of foveal depression, foveal thickening, vitreal displacement of foveal ONL, blurring or tenting of the foveal photoreceptor layer, and foveal detachment were all seen in eyes with worse BCVA (Figure 2). Our findings are consistent with speculations made by Gass, ${ }^{1}$ that the reduction in visual acuity caused by ERM may be related primarily to the distortion produced in the outer retinal layers and not by the size or degree of translucency of the ERM. Gass had used the term anterior herniation of the foveolar retina caused by contraction of the firmly adherent pericentral membrane. As the ONL contains cell bodies of the photoreceptors, changes in the ONL likely reflect the impact of traction on the foveal photoreceptors.

In addition to changes occurring in the outer retinal layers, FD-OCT imaging also allowed visualization of the changes in the inner retinal layers seen as striae (disarrangement) and irregularity in inner retinal layers with tethering of the NFL outside the fovea. These changes were seen more frequently in eyes with massive macular thickening and more severe vision loss. Whether these changes are secondary to massive macular edema or contribute directly to vision loss is unknown. We found a strong correlation between increase in MV and decrease in BCVA. Electrophysiological study of eyes with ERM have shown both inner and outer retinal dysfunction in eyes with vision loss from ERM. ${ }^{7}$

On the other hand, we found a poor correlation between macular thickening and decrease in visual function as assessed by MP-1, highlighting again the fact that retinal thickening per se outside the fovea does not necessarily result in reduced visual function. Similarly, Arichika $e t a l^{17}$ recently found that the inner retinal thickening in the perifoveal region did not correlate with decrease in visual acuity.

Reports have correlated the integrity of the photoreceptor IS-OS junction with visual function in various conditions. ${ }^{16-23}$ Recent publications using Stratus 
OCT by Suh et $a l^{5}$ and Mitamura et $a l^{6}$ reported a significant association between loss of integrity of the foveal photoreceptor IS-OS junction on OCT findings and visual acuity in eyes with ERM before and after vitrectomy surgery and correlated the integrity of the foveal photoreceptor IS-OS junction as a feature predictive of visual recovery after vitrectomy surgery in eyes with ERM. ${ }^{5,6}$ In our study, we found a poor correlation between photoreceptor IS-OS junction disruption seen on Stratus OCT and the photoreceptor IS-OS junction appearance on FD-OCT, showing the limitations of Stratus OCT in detecting abnormalities in the IS-OS junction especially in the presence of macular edema. In our careful review of all 24 eyes imaged with our high-resolution FD-OCT, there was no disruption of the photoreceptor IS-OS junction seen in any of the eyes with idiopathic ERM. However, a majority of the eyes with decreased BCVA had abnormalities in the foveal photoreceptor IS-OS junction, including an increase in foveal convexity of the IS-OS junction and blurring on the IS-OS junction and/or underlying VM which may appear as disruptions in foveal photoreceptor IS-OS junction with lower resolution OCT instruments.

The hyperreflective line just deep to the IS-OS junction, the VM, is thought to represent the interface between the photoreceptor outer segment tips and the villi of the retinal pigment epithelium. An increase in convexity of the foveal IS-OS junction is likely a manifestation of traction on the foveal photoreceptor cells. Blurring of the foveal IS-OS junction and the underlying VM noted in a majority of eyes with vision loss from idiopathic ERM likely represents misalignment or disorganization of the foveal photoreceptor cells from traction. In support of this theory is a recent case report, which detected fine microstriae among the macular cones using adaptive optics in an eye with idiopathic ERM and vision loss. The microstriae resolved completely after surgical removal of ERM along with complete visual recovery although macular morphology on OCT did not normalize. ${ }^{24}$

There are several limitations of the study including limited number of subjects enrolled and lack of postoperative imaging and/or histological correlate. This is because patients with BCVA worse than 20/60 often had other concurrent pathology contributing to vision loss which precluded enrollment in this study. As such, most of our enrolled subjects did not undergo vitrectomy surgery for removal of ERM. Nonetheless, our findings provide some insight to our understanding of the mechanism for the pathogenesis of vision loss associated with ERM and the potential usefulness of FD-OCT in evaluating patients with ERM. Now that commercially available FD-OCT instruments are used widely in clinical practice with image resolution close to the FD-OCT instrument used in this study (ie, axial resolution 5 to $5.5 \mu \mathrm{m}$ vs $4.5 \mu \mathrm{m}$ ), the findings of this study are more clinically applicable. A larger investigation can be considered using FD-OCT to study the morphological changes, both before and after surgical removal of the ERM, to help clinicians study in more detail the macular morphological changes associated with ERM.

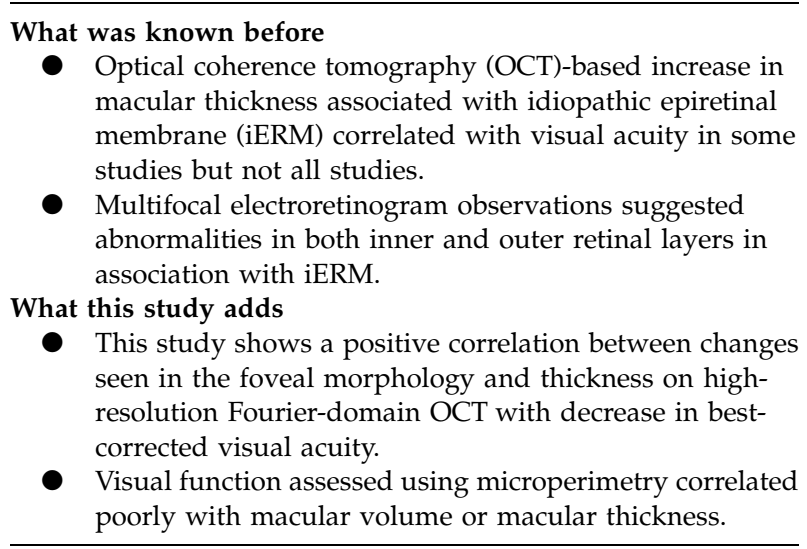

\section{Conflict of interest}

The authors declare no conflict of interest.

\section{Acknowledgements}

We acknowledge the help of Joseph Izatt from Bio-medical Engineering Department, Duke University, Durham, NC and Bioptigen Inc., Research Triangle Park, NC, for sharing real-time FD-OCT acquisition software. We also thank the members of Vision Science and Advanced Retinal Imaging laboratory, VSRI, and the institute for data analysis and visualization, IDAV, Department of Computer Science, University of California, Davis, for their help. This study was supported in part by Research to Prevent Blindness, New York, NY and the National Eye Institute, Bethesda, MD (Grant \#014743, JSW)

\section{References}

1 Gass JDM. Macular Dysfunction Caused by Vitreous and Vitreoretinal Interface Abnormalities. Stereoscopic Atlas of Macular Diseases, 4th edn. CV Mosby: St Louis, MO, 1996; 938-951.

2 McDonald HR, Johnson RN, Ai E, Schatz H. Macular Epiretinal membranes. In: Ryan SJ, Hinton DR, Schachat AP, Wilkinson P (eds). Retina, 4th edn, Vol 3, Mosby Elsevier Publishers, 2006, pp 2509-2525.

3 Wilkins JR, Puliafito CA, Hee MR, Duker JS, Reichel E, Coker JG et al. Characterization of epiretinal membranes 
using optical coherence tomography. Ophthalmology 1996; 103: 2142-2151.

4 Massin P, Allouch C, Haouchine B, Metge F, Paques M, Tangui L et al. Optical coherence tomography of idiopathic macular epiretinal membranes before and after surgery. Am J Ophthalmol 2000; 130: 732-739.

5 Suh MH, Seo JM, Park KH, Yu HG. Association between macular findings by optical coherence tomography and visual outcomes after epiretinal membrane removal. Am J Ophthalmol 2009; 147: 473-480.

6 Mitamura Y, Hirano K, Baba T, Yamamoto S. Correlation of visual recovery to presence of photoreceptor inner/outer segment junction in optical coherence images after epiretinal membrane surgery. Br J Ophthalmol 2009; 93: 171-175.

7 Parisi V, Coppe AM, Gallinaro G, Stirpe M. Assessment of macular function by focal electroretinogram and pattern electroretinogram before and after epimacular membrane sugery. Retina 2007; 27: 312-320.

8 Wojtkiowski M, Leitgeb R, Kowalczyk A, Bajraszewski T, Fercher AF. In vivo human retinal imaging by Fourier domain optical coherence tomography. J Biomedic Opt 2002; 7: 457-463.

9 Alam S, Zawadzki RJ, Choi S, Gerth C, Park SS, Morse L et al. Clinical application of rapid serial fourier-domain optical coherence tomography for macular imaging. Ophthalmology 2006; 113: 1425-1431.

10 Gloesmann M, Hermann B, Schubert C, Sattmann H, Ahnelt PK, Drexler W. Histological correlation of pig retina radial stratification with ultrahigh-resolution optical coherence tomography. Invest Ophthalmol Vis Sci 2003; 44: 1696-1703.

11 Zawadzki RJ, Fuller AR, Wiley DF, Hamann B, Choi SS, Werner JS. Adaptation of a support vector machine algorithm for segmentation and visualization of retinal structures in volumetric optical coherence tomography data sets. J Biomed Opt 2007; 12(4): 041206-1-041201-8.

12 Mirza RG, Johnson MW, Jampol LM. Optical coherence tomography use in evaluation of the vitreo-retinal interface: a review. Surv Ophthalmol 2007; 52: 397-421.

13 Zawadzki RJ, Jones SM, Oliver SS, Zhao M, Bower BA, Izatt JA et al. Adaptive-optics optical coherence tomography for high-resolution and high speed 3D retinal in-vivo imaging. Optics Express 2005; 13(21): 8532-8546.
14 Rasband WS. Image J. US National Institute of Health: Bethesda, MD. http://rsb.info.nih.gov/ij, 1997-2006.

15 Curcio CA, Sloan KR, Kalina RE, Hendrickson AE. Human photoreceptor topography. J Comp Neurol 1990; 292: 497-523.

16 Smith AJ, Telander DG, Zawadzki RJ, Choi SS, Morse LS, Werner JS et al. High-resolution fourier-domain optical coherence tomography and microperimetric findings after macula-off retinal detachment repair. Ophthalmology 2008; 115: 1923-1929.

17 Arichika S, Hangai M, Yoshimura N. Correlation between thickening of the inner and outer retina and visual acuity in patients with epiretinal membranes. Retina 2004; 30: 503-508.

18 Witkin AJ, Ko TH, Fujimoto JG, Chan A, Drexler W, Schuman JS et al. Ultra-high resolution optical coherence tomography assessment of photoreceptors in retinitis pigmentosa and related diseases. Am J Ophthalmol 2006; 142: 945-952.

19 Piccolino FC, de la Longrais RR, Ravera G, Eandi CM, Ventre L, Abdollahi A et al. The foveal photoreceptor layer and visual acuity loss in central serous chorioretinoapthy. Am J Ophthalmol 2005; 139: 87-99.

20 Inoue M, Watanabe Y, Arakawa A, Sato S, Kobayashi S, Kadonosono K. Spectral-domain optical coherence tomography images of inner/outer segment junctions and macular hole surgery outcomes. Graefes Arch Clin Exp Ophthalmol 2009; 247: 325-330.

21 Ota M, Tsujikawa A, Murakami T, Sato S, Kobayashi S, Kadonosono K. Association between integrity of foveal photoreceptor layer and visual acuity in branch vein occlusion. Br J Ophthalmol 2007; 91: 1644-1649.

22 Chang LK, Koizumi H, Spaide RF. Disruption of the photoreceptors inner segment-outer segment junction in eyes with macular holes. Retina 2008; 28: 969-975.

23 Oster FS, Mojana F, Brar M, Yuson RM, Cheng L, Freeman WR. Disruption of the photoreceptor inner segment/outer segment layer on spectral-domain optical coherence tomography is a predictor of poor visual acuity in patients with epiretinal membrane. Retina 2010; 30(5): 713-718.

24 Park SS, Choi SS, Zawadzki RJ, Werner JS. Fine retinal striae associated with epiretinal membrane visualized using adaptive optics. Retinal Cases Brief Rep 2009; 3: 233-237. 